\title{
Software for hydraulic design of axial-flow pump impellers and its manufacturing documentations
}

\author{
László Kalmár ${ }^{1 *}$, György Hegedűs ${ }^{2}$, Arpád Fáy ${ }^{1}$, and Norbert Szaszák ${ }^{1}$ \\ ${ }^{1}$ Institute of Energy Engineering and Chemical Machinery, Department of Fluid and Heat \\ Engineering, University of Miskolc, H-3515 Miskolc, Hungary \\ ${ }^{2}$ Institute of Machine Tools and Mechatronics, Department of Machine Tools, University of Miskolc, \\ H-3515 Miskolc, Hungary
}

\begin{abstract}
This article presents a hydraulic design procedure for axial-flow pump impellers, followed by their manufacturing documentations, all in one easy-to-use software named AXPHD V2.0 (AXial Pump Hydraulic Design) developed by one of the authors (Kalmár). After the user determined pump duty, the software offers input data which may be changed interactively. The hydrodynamic singularity method is used to compute the blade profiles on cylindrical surfaces. If the velocity and pressure distributions are accepted, then the body model of the impeller is produced by AUTODESK INVENTOR PROFESSIONAL 2019. Full manufacturing documentation is prepared including shop-drawings for traditional production, numeric modules for CAM, and files for 3D printing. A photo of an impeller made by $3 \mathrm{D}$ printing closes the paper.
\end{abstract}

\section{Introduction}

This paper has been written in an attempt to combine the hydraulic design procedure of axialflow pump impellers with the preparation of their laborious production documentation within one easy-to-use software package.

There are many pump makers who use their well-proven techniques for the design and manufacturing of their pumps. However, new problems may arise, new techniques may appear, and so even the oldest firms face changes. It may happen, for example, that for a new duty, pumps are required not with top efficiency, but with extra good cavitation performance, and with very safe operation for about 30 years. In coping with such extra cases, the designer may wish to have a new impeller design within hours. The software named AXPHD V2.0 developed by one of the authors may fulfil this task.

The package is also applicable for jobs in common industrial use e.g., for low head irrigation or drainage pumps.

The software is not suitable for pumps of extra high efficiencies since these require special research. However, the software may provide help even in such cases as an auxiliary tool reducing the time.

\footnotetext{
* Corresponding author: aramka@uni-miskolc.hu
} 
Special application of the software emerges in teaching. Getting familiar with the software the student meets all important variables of pump design, and producing a design is a concise job involving both hydraulic and manufacturing aspects.

\section{Computation model}

The main data of an axial-flow impeller are shown on Fig. 1. Outer diameter of the blades is $D_{K}$, the inner (hub) diameter $D_{B}$. Impeller speed is constant $n$, and the volume flow rate through the pump is $Q$.

A flow channel $F$ is shown in Fig. 2 with middle surface of radius $r$. The blade profile (to be designed) is also shown with absolute velocities $c_{F \sigma}(\sigma, \varphi)$ and $c_{F \varphi}(\sigma, \varphi), \sigma$ is axial coordinate, and $\varphi$ is angle measured from the inlet edge of one blade. Incompressible, frictionless flow, with zero radial velocity is assumed in the flow channel. The blade cascade (at radius $r$ ) evolved onto a plane is seen in Fig. 3 with the velocity triangles $\left(r, \sigma_{L}, L, T\right.$ in metres, $\mathbf{u}, \mathbf{c}, \mathbf{w}$ in $\mathrm{m} / \mathrm{s}$ ). The computations are executed for the flow through these blade cascades using the singularity method $[1,4-7]$.
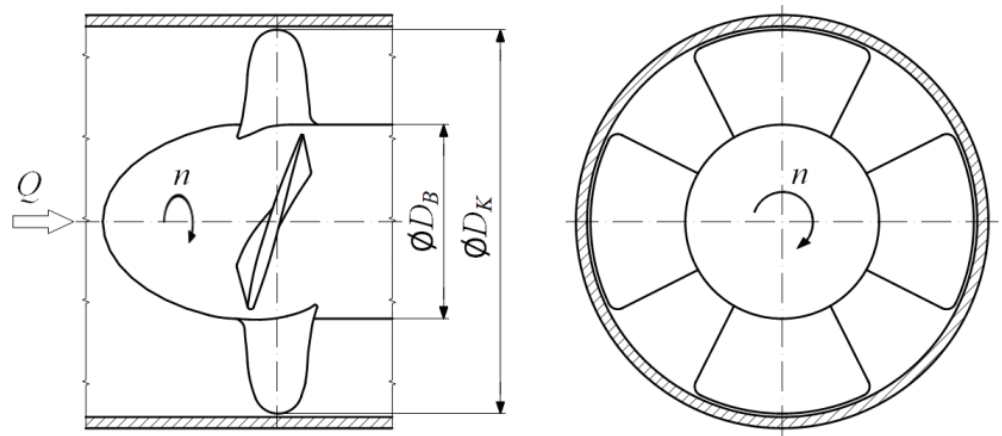

Fig. 1. Axial-flow pump, main data

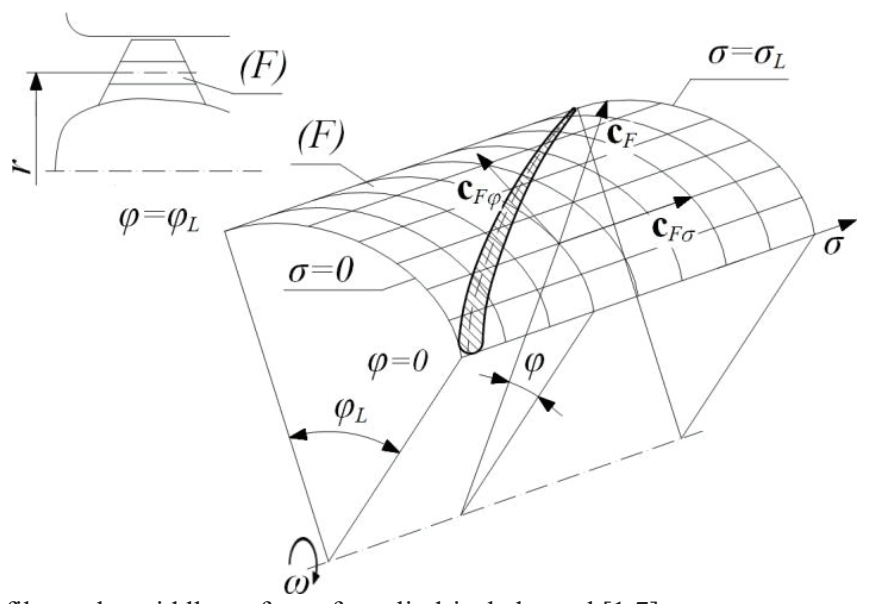

Fig. 2. Blade profile on the middle surface of a cylindrical channel $[1,7]$ 

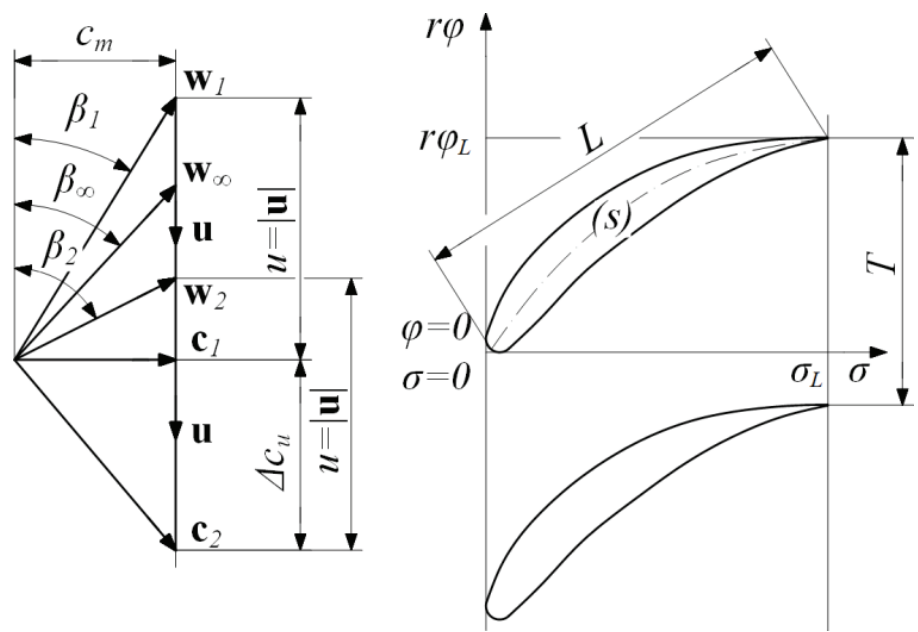

Fig. 3. Blade cascade evolved onto a plane, and the velocity triangles ( $\mathbf{u}$ is peripheral velocity of the blade cascade at radius $r, \mathbf{c}$ and $\mathbf{w}$ are the absolute and relative velocities of the fluid, 1 at inlet and 2 at outlet respectively).

\section{Main data}

The main operating parameters of the axial-flow pump to be designed are:

- $H$ : head of the pump $[\mathrm{m}]$,

- $Q$ : volumetric flow of the pump $\left[\mathrm{m}^{3} / \mathrm{s}\right]$,

- $n$ : speed of the pump [rpm],

- $\rho$ : density of the delivered fluid $\left[\mathrm{kg} / \mathrm{m}^{3}\right]$,

- and the specific speed calculated therefrom is: $n_{q}=n \frac{\sqrt{Q}}{\sqrt[4]{H^{3}}}$.

Many axial-flow pumps work in practice with wide operating ranges. However, in order to simplify the design, the pump is associated with one single set of the above parameters called nominal or design parameters. The success of a new design depends largely on suitable selection of these parameters.

The usual head and discharge ranges of axial-flow pumps are:

$$
2.5 \mathrm{~m} \leq H \leq 10 \mathrm{~m} \text {, and } 0.01 \frac{\mathrm{m}^{3}}{\mathrm{~s}} \leq Q \leq 10 \frac{\mathrm{m}^{3}}{\mathrm{~s}} .
$$

Of course, there are known units operating at $H=20 \mathrm{~m}$, but these are exceptional. The fluid is clean or raw water: $\rho=1000 \mathrm{~kg} / \mathrm{m}^{3}$. Some tender documents limit speed $n$ because fear from excessive cavitation, and in selecting nominal data this must be respected. If $n$ has not been fixed, using specific speed $n_{q}$ is advised to be selected not too far from current industrial practice (Table 1):

Table 1. Suggestion for specific speed

\begin{tabular}{|l|c|c|c|c|c|c|}
\hline$H$, meter & 3 & 4 & 5 & 6 & 7 & 8 \\
\hline$n_{q}$, metric & 330 & 270 & 230 & 200 & 170 & 140 \\
\hline
\end{tabular}

Other design parameters are suggested by the software in Figs 4, 5, 6, based on industrial practice. 


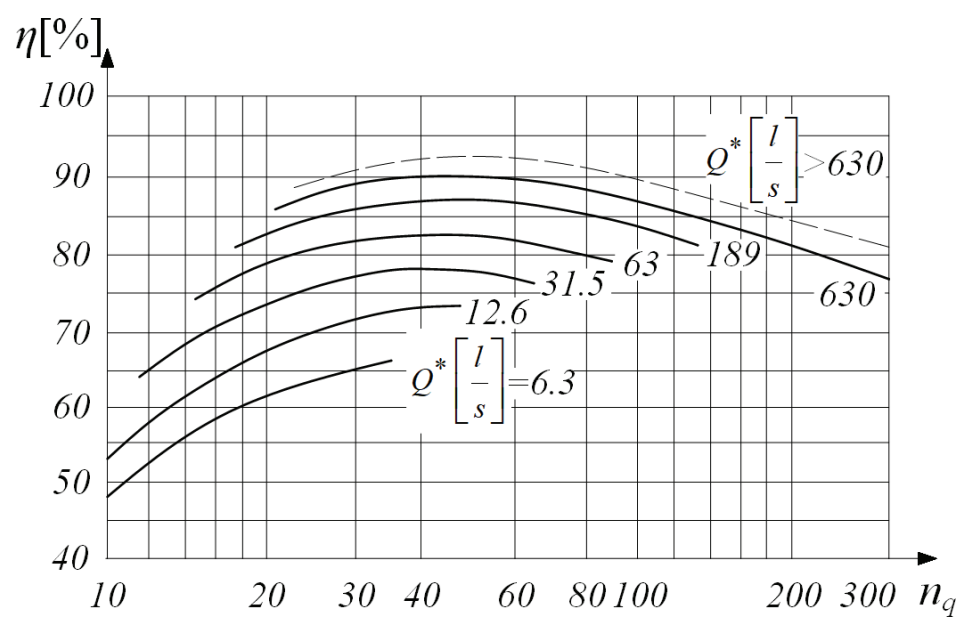

Fig. 4. Efficiency of the pump bowl [3]

The pump bowl includes impeller and guide vane row ( $\eta$ is pump efficiency, but without the loss of the discharge bend). The inlet flow coefficient $\varepsilon$ is defined by: $c_{m}=\varepsilon \sqrt{2 g H}$, where $c_{m}$ is meridional flow velocity upstream of the blades. The outer diameter of the impeller $D_{K}$ is calculated from $Q=\frac{\left(D_{K}^{2}-D_{B}^{2}\right) \pi}{4} c_{m}$, while the hub diameter $D_{B}$ accords with Fig. 6.

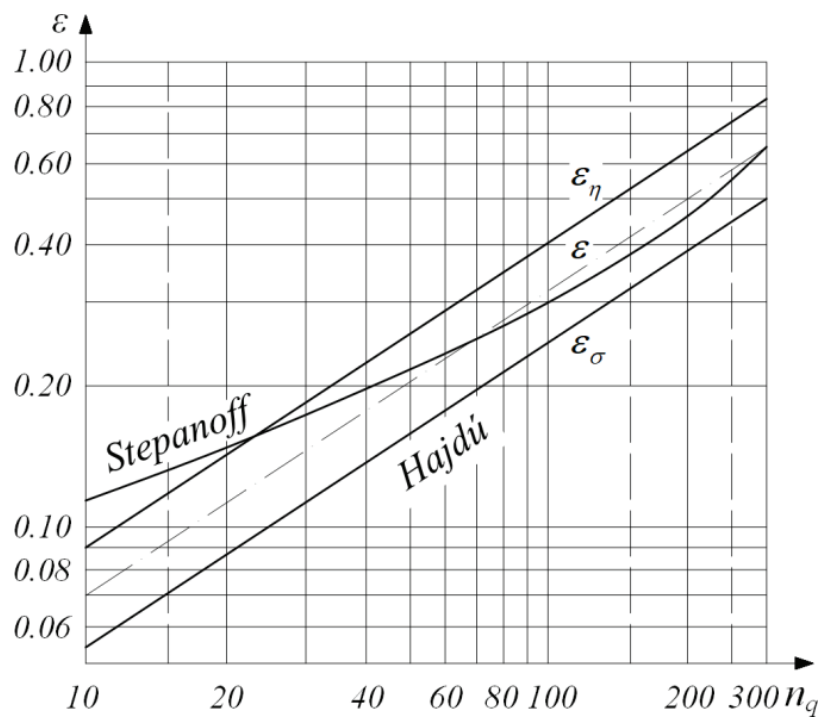

Fig. 5. Inlet flow coefficient [3], Stepanoff [8], Hajdú [9]

Figure 5 is used for suitable selection of $\varepsilon$. Using $\varepsilon_{\eta}$ is recommended to achieve good efficiency, while $\varepsilon_{\sigma}$ to reach good cavitation conditions, but the user may choose naturally another $\varepsilon$ value. 


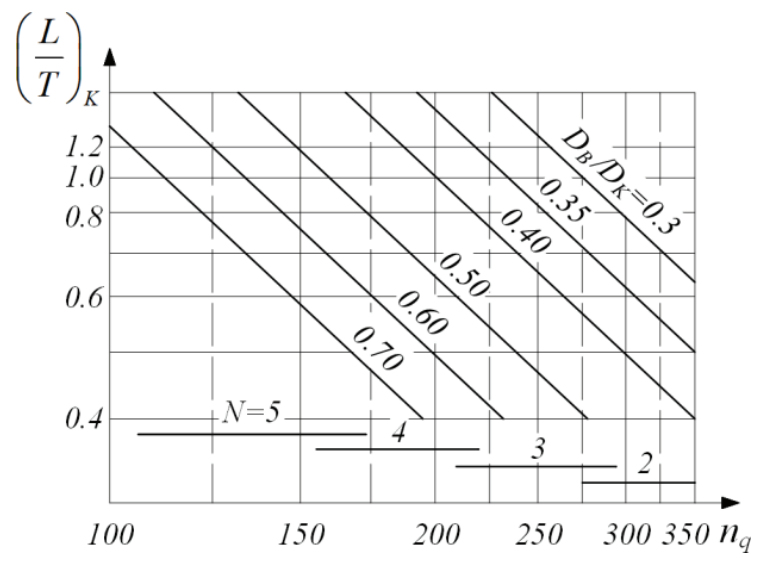

Fig. 6. Main geometric data of the impeller ( $N$ is number of blades) [3]

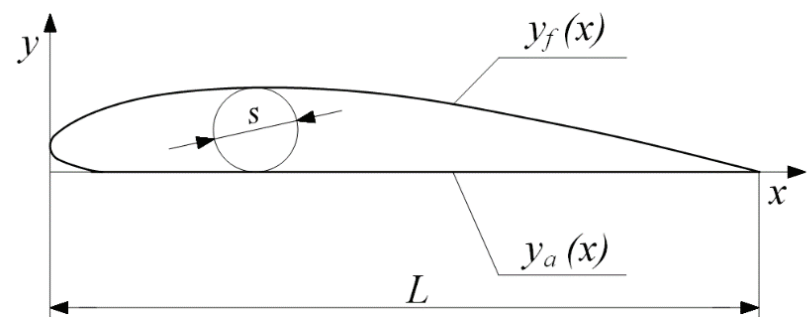

Fig 7. Profile section with maximum thickness

The software offers also values for $\left(\frac{L}{T}\right)_{B},\left(\frac{s}{L}\right)_{K},\left(\frac{s}{L}\right)_{B}$ (Fig. 7). The peripheral velocity is: $u=$ $r \omega$, and $\Delta c_{u}$ (Fig. 3) is calculated from: $u \Delta c_{u}=\frac{g H}{\sqrt{\eta}}$.

\section{Computation of flow}

For the flow (Fig. 2) conservation of mass and circulation-free conditions are required:

$$
\begin{aligned}
& \frac{\partial c_{F \sigma}}{\partial \sigma}+\frac{1}{r} \frac{\partial c_{F \varphi}}{\partial \varphi}=0 \\
& \frac{\partial c_{F \varphi}}{\partial \sigma}+\frac{1}{r} \frac{\partial c_{F \sigma}}{\partial \varphi}=0 .
\end{aligned}
$$

The partial differential equation system (Equs. (1) - (2)) together with the

$$
\sigma=0: c_{F \sigma}=c_{F 1 \sigma}=c_{m} ; c_{F \varphi}=c_{F 1 \varphi}=0
$$

and

$$
\sigma=\sigma_{L}: c_{F \sigma}=c_{F 2 \sigma}=c_{m} ; c_{F \varphi}=c_{F 2 \varphi}=\Delta c_{u}(r)
$$

boundary conditions is available to determine the unknown functions of $c_{F \sigma}(\sigma, \varphi)$ and $c_{F \varphi}$ $(\sigma, \varphi)$ used to describe the velocity distributions along the middle surface $(F)$ (see Fig. 2). 
The middle surface of the flow channel (Fig. 2) evolved onto a plane, results in a straight blade cascade (Fig. 3) where the velocities have the same sizes as in Fig. 2. The camber lines of the profiles are computed (rendering ideal incidence flow to the impeller in pump nominal operating point). The singularities (vortex and source-sink distributions) are then placed on the camber line. The code offers a few distributions which the user may select. Thus, all parameters needed for the design are known, and the computations using the singularity method are carried out. The contour points obtained for the blades are then available, and non-dimensional velocity and pressure distributions are shown for approval. If accepted, the blade coordinates are used to build up the solid model of the impeller as shown below.

The code assumes frictionless flow. It may happen that the user intends to make a loss analysis (not included in the code, but any user's program can easily be adjoined). For such analysis the computed velocity field is easily accessible.

\section{Software Modules}

The AXPHD V2.0 design software was developed in the frame of Microsoft Visual Studio Community Edition using the $\mathrm{C} \#$ language. The Code allows quick input, performs numerical algorithms, stores data, communicates to user written programs (Fortran, $\mathrm{C} / \mathrm{C}, \mathrm{C}++, \mathrm{VB}$, Java), and prepares many things for manufacturing the impeller. The Main menu consist of the following modules:

- Project management,

- Specification and definition of hydraulic and geometric characteristics,

- Implement the hydraulic design of the impeller,

- Development of the impeller's 3D body model,

- View and document the results.

Project management means selecting a new name or using one from the list of old names. Several impeller variants may be included in one project. Among others the following functions are used: Input, Save, Save as, Archive, Delete. The modules are left by pressing Back to Main menu.

Specification and definition of hydraulic and geometric characteristics. The module opens input Panels enabling easy identification of data. For impeller G162 (Fig. 11) designed for a model pump subject to hydraulic test, the following values were fed into Panel P1:

- Manometric head:

$H=4.4[\mathrm{~m}]$,

- Volumetric flow: $\quad Q=0.437\left[\mathrm{~m}^{3} / \mathrm{s}\right]$,

- Speed of rotation: $\quad n=1150[\mathrm{rpm}]$,

- Density of fluid: $\quad \rho=1000\left[\mathrm{~kg} / \mathrm{m}^{3}\right]$.

The specific speed calculated therefrom becomes $n_{q}=250\left[\mathrm{rpm}, \mathrm{m}^{3} / \mathrm{s}, \mathrm{m}\right]$. Other input data are fed using panels P2 - P7. After inputs are completed, output Panels may show the data. For G162 the main output parameters are: Number of blades: $N=3$, outer diameter: $D_{K}=0.35[\mathrm{~m}]$, inner diameter: $D_{B}=0.154[\mathrm{~m}]$. Using 7 different radius $r$ for the cylindrical sections has also been approved.

Implement the hydraulic design of the impeller. The singularity method computes the blade contours on the cylindrical sections only, and their final positions in the 3D space are determined as prescribed in the former module. Figure 8 shows the drawing of the hydraulic surfaces of the blade. 


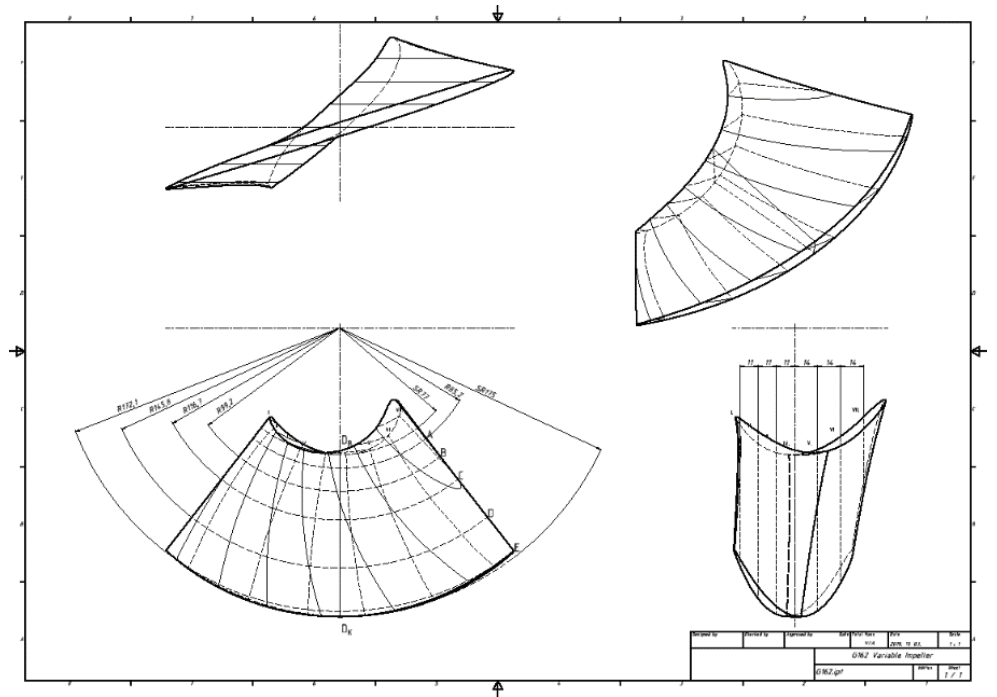

Fig. 8. Drawing of the blade hydraulic surfaces

Development of the impeller's $3 D$ body model. This module offers first a list of possible documentations for selection. The blade is completed with parts joining to the hub (Fig. 9 a), which is also treated as a body ready for making (Fig. 9 b).

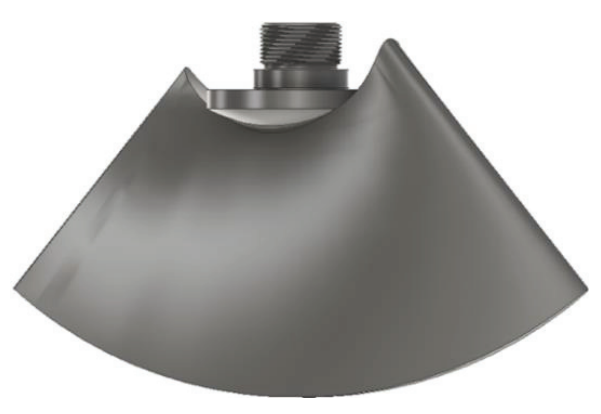

(a)

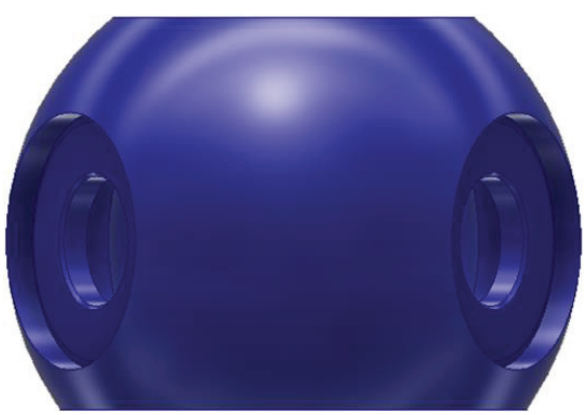

(b)

Fig. 9. View of the body model of the blade

View and document the results. This module offers saving for any activities defined in the modules. Various libraries may be opened, and various formats (docx, 2Dpdf, 3Dpdf, stp) may be used.

\section{Impeller produced by 3D printing}

Files of format stp are suitable for producing 3D printing. The impeller G162, designed for a model pump (Fig. 10) by AXPHD V2.0, has been produced using 3D printing with the help of the specialists from KVINT-R Ltd. The accuracy proved to be excellent, even the threads produced by $3 \mathrm{D}$ printing, which fix the blades in the hub, worked appropriately with commercial nuts. This model pump impeller has been prepared for testing in a cavitation test rig of $350 \mathrm{~mm}$ impeller diameter. 
The work outlined in this paper has been devoted to help pump design. The software is flexible, offers various options for general use, and moreover to these, major changes of the procedure may easily be introduced by user defined programs.

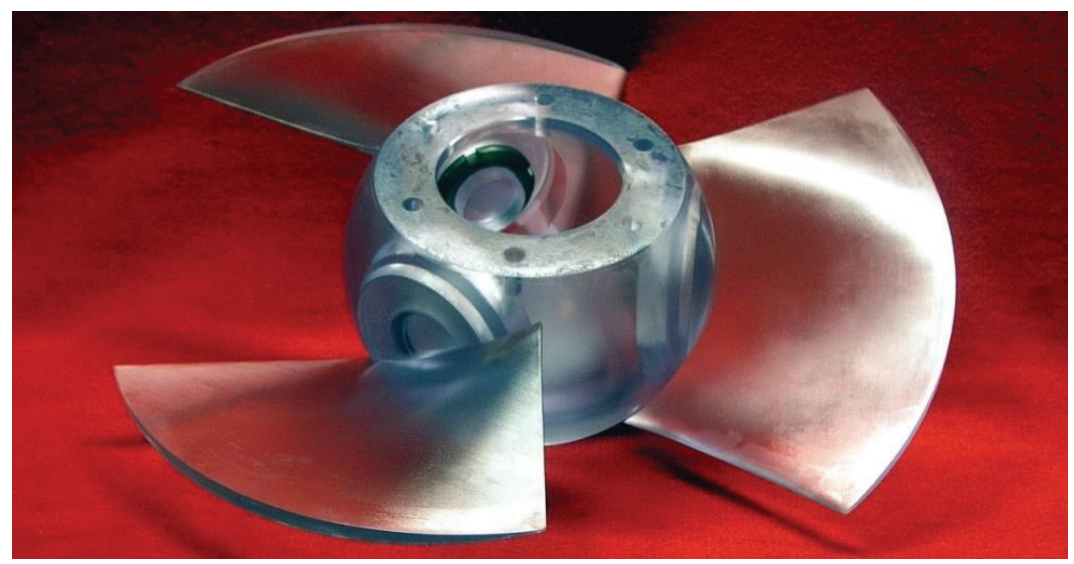

Fig. 10. Photo of axial-flow impeller designed by AXPHD V2.0 produced by 3D printing for model tests

This research was carried out as part of the GINOP-2.1.7-15-2016-00429 project with support by the European Union, co-financed by the European Social Fund.

\section{References}

1. Czibere, T.: The solution of the two main tasks of the hydrodynamic blade cascade computations by using the potential-flow theory (in Hungarian), Miskolc, (1965)., p. 154.

2. Czibere, T. - Kalmár L.: Softwares for turbomachines: solution of the first main task of cascade computations operating with compressible media (in Hungarian), Research report, Miskolc, (1975)., p. 54.

3. Czibere, T.: Turbomachines, lecture notes, (J14-500), Budapest, (1977).

4. Fáy, Á.: Simple performance model for pumps, ASME Symposium "Pumping Machinery 1993", Fluids Engineering Conference, Washington D.C. June 20-24. (1993).

5. Lewis, R.I.: Vortex element methods for fluid dynamic analysis of engineering systems, (2005), books.google.com.

6. Kalmár, L.: Computer aided design of axial pump impellers, Research report, Miskolc, (2014).

7. Kalmár, L. Hegedüs, G. Czibere, T.: Computer Aided Designing of Blading of the Axial Flow Pump Impeller, Pumps, Compressors, Vacuum Pumps, Hungary, vol. XXIII, no. 1, pp. 53-68, (2016).

8. Stepanoff, A.J.: Centrifugal and axial flow pumps, (1957), John Wiley and Sons.

9. Hajdú, S. Szalay, Gy.: Parameters for the qualifications of irrigation and drainage pumps, Papers on Water Management (1967), No. 4 